\title{
Solvent Vapor Annealing Effects in Contact Resistances of Zone-cast Benzothienobenzothiophene $\left(\mathrm{C}_{8}-\mathrm{BTBT}\right)$ Transistors
}

\author{
Chaewon Kim, Anjae Jo, Heeju Kim, Miso Kim*, Jaegab Lee, and Mi Jung Lee ${ }^{\dagger}$ \\ School of Advanced Materials Engineering, Kookmin University, Seoul 02707, Korea \\ ${ }^{*}$ Center for Safety Measurement, Korea Research Institute of Standards and Science (KRISS), Daejeon 34113, Korea
}

(Received July 2, 2016; Revised July 19, 2016; Accepted July 19, 2016)

\begin{abstract}
Benzothienobenzothiophene $\left(\mathrm{C}_{8}\right.$-BTBT) is a soluble organic small molecule material with high crystallinity resulting from its strong self-organizing properties. In addition, the high mobility and easy fabrication of $\mathrm{C}_{8}$-BTBT make it very attractive in terms of organic thin-film transistors. In this work, we made $\mathrm{C}_{8}$-BTBT thin films by using the zone-casting method; we also used an organic solvent to treat the devices with solvent vapor annealing to improve the electrical properties. As a result, we confirmed improved mobility, threshold voltage, and subthreshold swing after solvent vapor annealing. To prove the effect of solvent vapor annealing, we used the simultaneous extraction model to extract the contact resistance from the current-voltage curve. We confirmed that the electrical properties improved with decreasing contact resistance.
\end{abstract}

Key words : $C_{8}$-BTBT, Zone-casting, Solvent vapor annealing, Contact resistance, Organic field effect transistors (OTFTs)

\section{Introduction}

$\mathrm{M}$ any researchers have been focused on organic semiconductors over the past decades because these devices have many advantages such as low-cost, large-area deposition, and flexibility. ${ }^{1,2)}$ Recently, the field effect mobility of small-molecule organic semiconductors has exceeded that of amorphous silicon, which shows the potential for these devices to be applied to electronic products. ${ }^{3,4)}$ With modification of the molecular structure, many organic semiconductors have or can have good solubility in organic solvents. This solubility property enables organic materials to be utilized through solution process, which is a very attractive process compared to other techniques. ${ }^{5,6)}$ The solution process is cost effective because it does not require a vacuum or high temperature system; this allows simplified fabrication procedures compared to the conventional microfabrication process, which is based on vacuum technology. ${ }^{7,8)}$ Therefore, soluble organic materials have a strength in the realization of flexible electronic devices such as displays, thin-film transistors (TFTs), solar cells etc. Among the various solution processes, zone-casting ${ }^{9,10,11)}$ allows the fabrication of well-ordered organic thin films; zone-casting, due to the use of a specialized flat nozzle and a mobile substrate carrier, is more beneficial for large-area device fabrication than are other solution techniques such as inkjet printing,

${ }^{\dagger}$ Corresponding author : Mi Jung Lee

E-mail : mijung@kookmin.ac.kr

Tel : +82-2-910-4664 Fax : +82-2-910-4320 drop casting, ${ }^{12)}$ or spin coating. ${ }^{13)}$ In this paper, one soluble small molecule organic material, benzothienobenzothiophene $\left(\mathrm{C}_{8}\right.$-BTBT), was zone-cast; this material has high mobility in organic thin film transistors (OTFTs). ${ }^{14)}$ And, solvent vapor annealing (SVA ${ }^{15,16)}$ was conducted to enhance the performance by improving the charge injection properties of the OTFTs.

\section{Experimental Procedure}

Prior to the fabrication of zone-cast organic thin films, cleaned silicon substrates with 300 nm-thick $\mathrm{SiO}_{2}$ were used. The wafer was cleaned in piranha solution on a $100^{\circ} \mathrm{C}$ hotplate for $10 \mathrm{~min}$; the cleaning solution was composed of $\mathrm{H}_{2} \mathrm{SO}_{4}$ and $\mathrm{H}_{2} \mathrm{O}_{2}$ at a ratio of $7: 3$. Samples were then cleaned using an ultrasonic generator in acetone, IPA, and D.I water for $10 \mathrm{~min}$, each. To optimize the concentration of the $\mathrm{C}_{8}$-BTBT solution, $2,5,10$, and $15 \mathrm{mg} / \mathrm{ml}$ solution concentrations were adopted. $\mathrm{C}_{8}$-BTBT was dissolved in 1,2dicholorobenzene (DCB) and zone-casting was carried out at $5 \mathrm{~mm} / \mathrm{s}$ speed. All procedures were executed in nitrogen atmosphere. During the casting process, the solution temperature and the substrate temperature were maintained at $80^{\circ} \mathrm{C}$ and $120^{\circ} \mathrm{C}$, respectively. A $100 \mathrm{~nm}$ thick Au contact was deposited by thermal evaporation to make the source and drain and to fabricate the bottom-gated top-contact structure. The channel length and the width of the shadow mask were 50 and $2000 \mu \mathrm{m}$, respectively. Devices were treated with solvent vapor on the hotplate, and were then sealed in a glass bath for $1 \mathrm{~h}$ at ambient atmosphere. The surface morphology of the $\mathrm{C}_{8}$-BTBT thin film was observed 
(a)

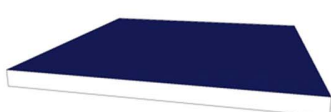

$\mathrm{SiO}_{2}(300 \mathrm{~nm}) / \mathrm{Si}$ substrate

(c)

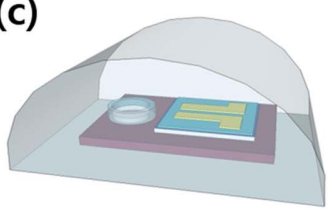

Solvent vapor annealing (SVA) (b)
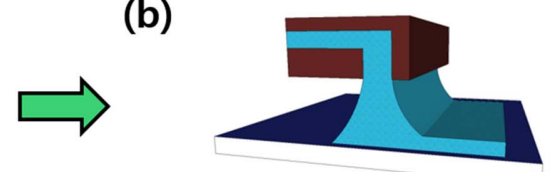

Zone casting of $\mathrm{C}_{8}$-BTBT solution

(d)

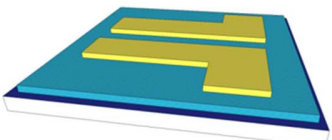

Au source \& drain deposition

Fig. 1. Schematic experimental procedures of $\mathrm{C}_{8}$-BTBT transistors. (a) Cleaning of $300 \mathrm{~nm} \mathrm{SiO}_{2}$ wafer, (b) zone casting to fabricate $\mathrm{C}_{8}$-BTBT thin films, (c) thermal evaporation of $100 \mathrm{~nm}$ Au source and drain, (d) solvent vapor annealing in ambient atmosphere.
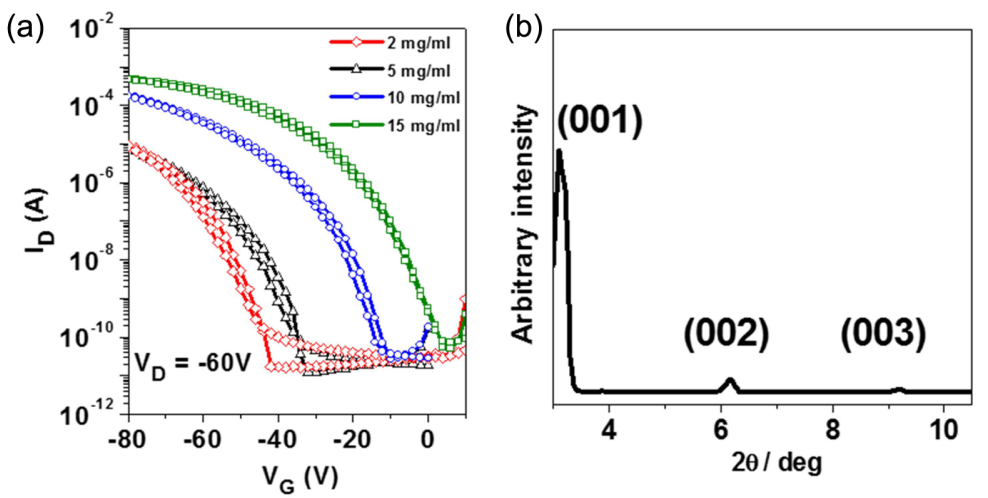

Fig. 2. (a) Transfer characteristics of $\mathrm{C}_{8}$-BTBT thin film transistors with various concentrations $(2,5,10,15 \mathrm{mg} / \mathrm{ml})$, (b) XRD measurements of one zone-cast $\mathrm{C}_{8}$-BTBT thin film.

by atomic force microscopy (AFM) (XE-100, Park System). We used X-ray diffraction (XRD) (D/Max-2500V, Rigaku) to confirm the crystalline nature of the $\mathrm{C}_{8}$-BTBT thin film. The electrical characteristic of the $\mathrm{C}_{8}$-BTBT transistors was measured using a semiconductor characterization system (Keithley 4200-SCS).

\section{Results and Discussion}

To fabricate highly oriented crystalline films, zone casting was conducted with various solution concentrations. Fig. 1 details the schematic experimental procedures of the $\mathrm{C}_{8}$-BTBT transistors fabricated by zone-casting method.

A heavily doped $n+$ silicon wafer with thermally oxidized silicon dioxide $\left(\mathrm{SiO}_{2}\right)(300 \mathrm{~nm})$ was used as a substrate. An optimized concentration of the $\mathrm{C}_{8}$-BTBT solution was used from 2 to $15 \mathrm{mg} / \mathrm{ml}$. Since the film morphology varies sensitively with the temperature (for both the substrate and the solution), and the casting speed, the zone-casting condition was established using a temperature of $80^{\circ} \mathrm{C}$ of the solution, and a temperature of $120^{\circ} \mathrm{C}$ of the substrate; the casting speed was $5 \mathrm{~mm} / \mathrm{s}$. Also, to prevent any possible degradation of the solution at elevated temperature by oxygen and moisture in the atmosphere, casting was carried out in a nitrogen-filled glove box. After the casting process, the films were heated at $100^{\circ} \mathrm{C}$ on the hot plate to eliminate the remaining solvent of $\mathrm{C}_{8}$-BTBT thin film. For the process of solvent vapor annealing, samples were heated to $80^{\circ} \mathrm{C}$ on the hotplate with a small amount of DCB (b.p. $180^{\circ} \mathrm{C}$ ) in a sealed glass bath. Solvent vapor annealing was performed for $1 \mathrm{~h}$.

Transfer curves with various concentrations of $\mathrm{C}_{8}$-BTBT solution are shown in Fig. 2(a). As the concentration of the $\mathrm{C}_{8}$-BTBT solution increased, the turn-on voltage moved from negative bias to zero without change of the off-current; this change is related to the microstructure of the molecular stacking in the cast film. We determined that $15 \mathrm{mg} / \mathrm{ml}$ is the optimized solution concentration, and this concentration 
(a)

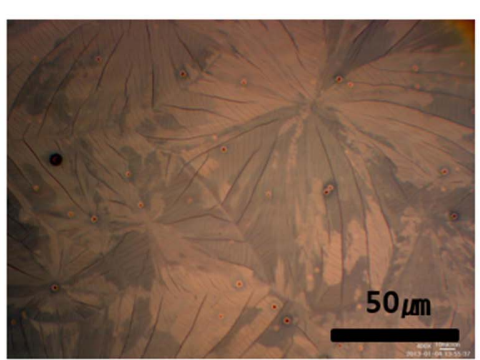

(c)

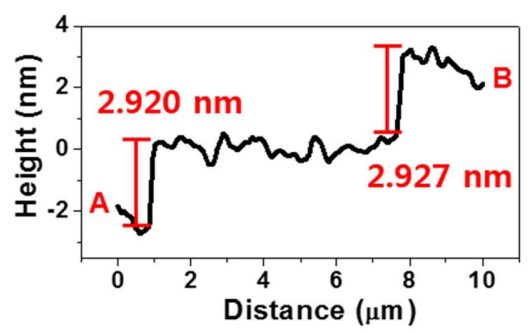

(b)

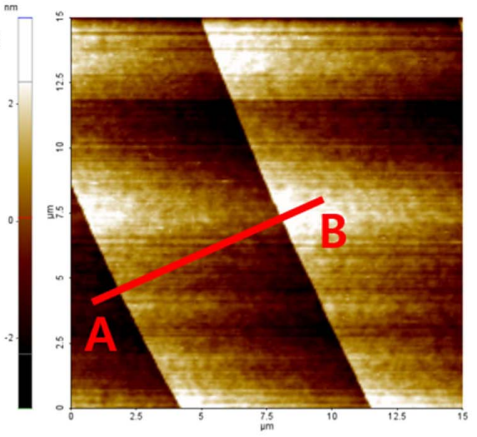

(d)

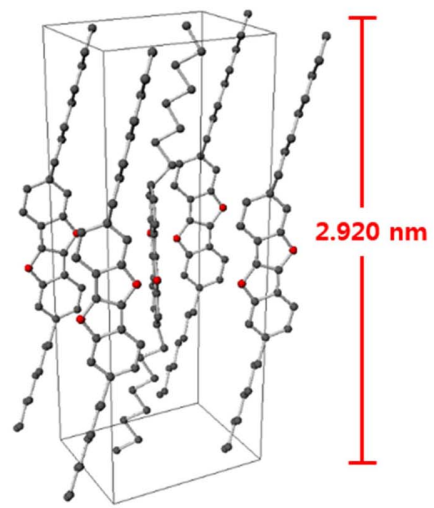

Fig. 3. Characterization of $\mathrm{C}_{8}-\mathrm{BTBT}$ thin film. (a) Optical microscope image of zone-cast thin film, (b) atomic force microscopy (AFM) image showing morphology of the surface, (c) line spectrum extracted from red line in (b), (d) schematic of molecular arrangement.

was used to fabricate the $\mathrm{C}_{8}$-BTBT FETs.

$\mathrm{XRD}$ analysis shows a well-ordered $\mathrm{C}_{8}$-BTBT thin film along the $c$-axis, as can be seen in Fig. 2(b). Three peaks were detected at $3.1,6.15$, and $9.2^{\circ}$, which indicated the (001), (002), and (003), respectively. The lattice structure, which is related to the XRD data, will be discussed later, along with a discussion of the molecular stacking of the $\mathrm{C}_{8}$ BTBT.

Figure 3(a) is an optical microscope image of the $\mathrm{C}_{8}$-BTBT thin film with large crystal. A snowflake-shape was observed; there was no specific orientation of the crystalline with respect to the zone casting direction in this work. The isotropy of the cast film is due to the high speed of casting; an aligned film along the casting direction was observed when the casting speed was slow $(0.4 \mathrm{~mm} / \mathrm{s}$; data is not shown here).

Surface morphology was observed by AFM and is shown in Fig. 3(b). ${ }^{17)}$ The $\mathrm{C}_{8}$-BTBT thin film shows two large and apparent terraces within an area of $15 \times 15 \mu^{2}$. The height of the terrace was measured by checking the line spectrum from the red line (A-B) in Fig. 3(b). The height of the terrace was approximately $2.92 \mathrm{~nm}$, which corresponds to the length of the $c$-axis of the $\mathrm{C}_{8}$-BTBT monolayer in the crystal structure. The molecular arrangement of $\mathrm{C}_{8}$-BTBT is depicted in Fig. 3(d), with the length of the $c$-axis.

Before and after solvent vapor annealing, the electrical characteristics of the transistors were measured under high vacuum $\left(\sim 10^{-6}\right.$ torr). Fig. 4(a) and 4(b) show the transfer characteristics and the square root of the drain current in the saturation regime along the gate voltage. Drain current was measured at linear $\left(V_{\mathrm{D}}=-5 \mathrm{~V}\right)$ and saturation regimes $\left(V_{\mathrm{D}}=-60 \mathrm{~V}\right)$. Before solvent vapor annealing, the mobility was $0.32 \mathrm{~cm}^{2} / \mathrm{Vs}$; the subthreshold voltage $\left(V_{\mathrm{th}}\right)$ and the subthreshold swing (SS) were $-22 \mathrm{~V}$ and $7 \mathrm{~V} /$ decade, respectively, in the saturation regime. After solvent vapor annealing, the mobility, subthreshold voltage, and subthreshold swing were $0.35 \mathrm{~cm}^{2} / \mathrm{Vs},-12 \mathrm{~V}$, and $1.5 \mathrm{~V} /$ decade, respectively. After solvent vapor annealing, the main factors of the TFT performance, such as the mobility, threshold voltage, and subthreshold swing, were found to have improved. Fig. 4(c) and 4(d) indicate the output curves before and after solvent vapor annealing, respectively. Comparing Fig. 4(c) and 4(d), we found that the maximum drain current increased after SVA, which leads to higher mobility. Also, suppression of the drain current, indicated by the linear ohmic curve, at small drain bias was reduced after solvent vapor annealing; this indicates a more facile charge injection from the metal contact electrode to the semiconducting layer. We believe that the enhancement of the device characteristics with solvent vapor annealing is caused by reduced contact resistance between the $\mathrm{C}_{8}$-BTBT layer and the Au contact electrodes.

To investigate the effect of solvent vapor annealing, we calculated the contact resistance using a comparison of $\mathrm{C}_{8}$ - 
(a)

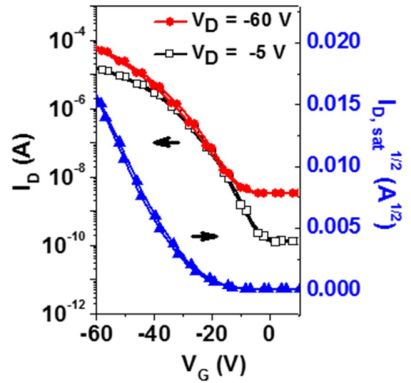

(c)

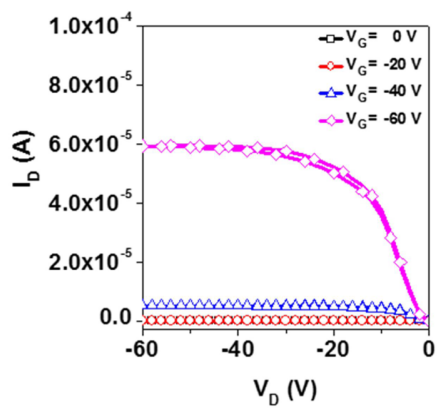

(b)

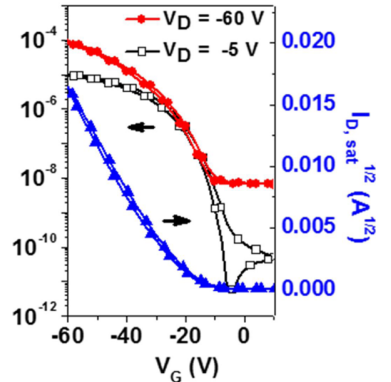

(d)

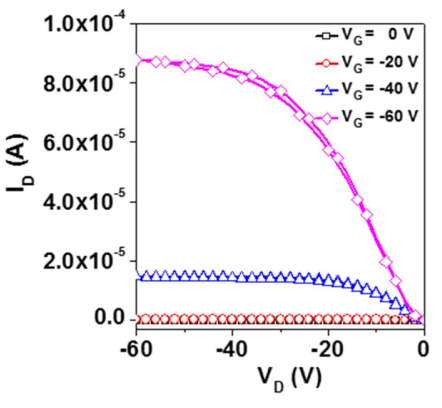

Fig. 4. Electrical characteristics of $\mathrm{C}_{8}$-BTBT TFTs. (a) and (b) indicate transfer curves before and after SVA. The black square indicates the linear region and the red circle indicates the saturation region. The blue triangle denotes the square root of the drain current. Output curves are in (c) and (d), before and after SVA, respectively, as a function of gate voltage.
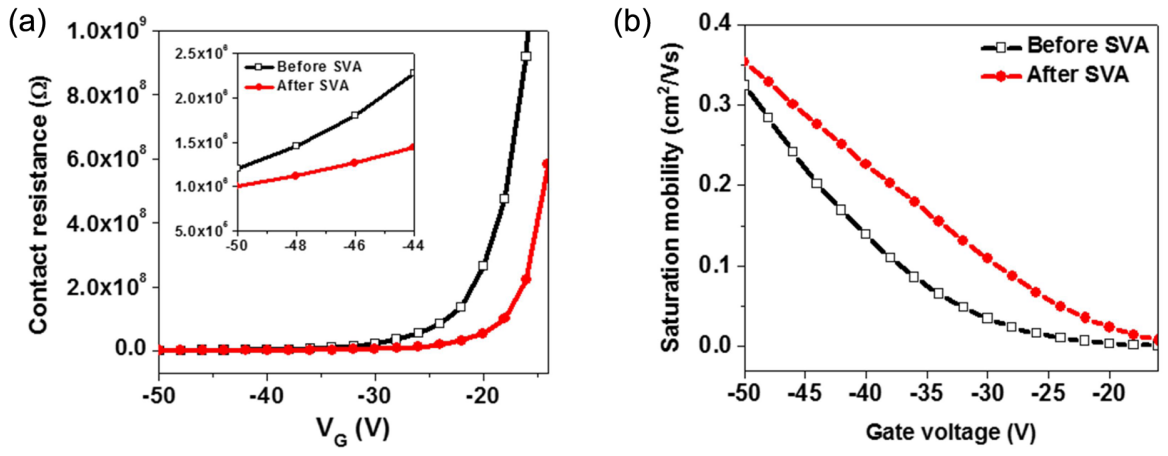

Fig. 5. (a) Contact resistances as function of gate voltage; graph was extracted using the simultaneous extraction model. Inset provides magnification of the graph. (b) Saturation mobilities both before and after SVA.

BTBT devices before and after solvent vapor annealing. Contact resistance was directly estimated as a function of the gate voltage using the transfer curves; this method is the simultaneous extraction model suggested by di Pietro et al. ${ }^{18,19)}$

$$
\begin{aligned}
& \frac{\frac{I_{\text {lin }}}{d V_{G}} \cdot k}{2\left(\frac{d \sqrt{I_{\mathrm{sat}}}}{d V_{\mathrm{G}}}\right)^{2}} \cong V_{\mathrm{D}^{*} \mathrm{~S}}\left(V_{\mathrm{G}}\right) \\
& R_{\mathrm{C}}\left(V_{\mathrm{G}}, V_{\mathrm{DS}}\right)=\frac{\left(V_{\mathrm{DS}}-V_{\mathrm{D}^{* \mathrm{~S}} *}\left(V_{\mathrm{G}}\right)\right)}{I_{\mathrm{D}}^{\text {lin }}}
\end{aligned}
$$

$V_{\mathrm{D}^{*} \mathrm{~S}^{*}}$, the channel voltage drop, was determined from Eq. (1), in which $k$ was obtained using power laws, $\left[\mu\left(V_{\mathrm{G}}\right)=\right.$
$\left.\mathrm{a}\left(V_{\mathrm{G}}\right)^{\mathrm{B}}\right]$ and $k=(B+2) /(2(B+1))$, which is commonly used for the estimation of the charge density dependent mobility. The contact resistance was extracted using Eq. (2) once $V_{\mathrm{D}^{*} \mathrm{~S}^{*}}$ had been obtained from the transfer curves of the linear and saturation regimes.

A comparison of the contact resistances before and after solvent vapor annealing, according to the gate bias extracted from the equations above, is shown in Fig. 5(a). A definite reduction in the contact resistance can be observed after solvent vapor annealing. Especially, the difference of the contact resistance at small gate bias, at which the charge injection properties are critical for device performance, was distinct. This can provide an explanation for the remarkable enhancement of the subthreshold voltages and the subthreshold swing properties evident in the transfer curves after solvent vapor treatment. The difference of the contact resis- 
tance becomes smaller as the gate voltages increase; the contact resistance values were $1.2 \times 10^{6} \Omega$ and $1.0 \times 10^{6} \Omega$ before and after solvent vapor annealing at $-50 \mathrm{~V}$ of gate voltage, respectively, as can be seen in inset in Fig. 5(a), which is a magnified plot of Fig. 5(a) in a range from $-44 \mathrm{~V}$ to $-50 \mathrm{~V}$. Saturation mobility also increased from $0.32 \mathrm{~cm}^{2} / \mathrm{Vs}$ to 0.35 $\mathrm{cm}^{2} / \mathrm{Vs}$, a relatively small amount considering the improvements of the subthreshold voltages and the subthreshold swing properties. These improvements seem to be related with the similar contact resistance values at higher gate voltages. We suggest that solvent vapor annealing of the $\mathrm{C}_{8}$ BTBT transistors was effective at reducing the contact resistance but did not affect the $\mathrm{C}_{8}$-BTBT channel properties due the annealing temperature, which was too low to induce recrystallization of the molecules. During the process of solvent vapor annealing, relief of the strain that accumulated between the $\mathrm{Au}$ contact and the $\mathrm{C}_{8}$-BTBT films during thermal evaporation can be seen as a reason for the improved charge injection. Therefore, solvent vapor annealing can be utilized as a useful post-treatment to reduce contact resistance via a simple process, while intrinsic film properties are not affected.

\section{Conclusions}

We fabricated $\mathrm{C}_{8}$-BTBT transistors by zone-casting technique. The basic characteristics of $\mathrm{C}_{8}$-BTBT thin film were observed by optical microscopy, AFM, and XRD; the film showed a well-ordered large crystalline structure along the (001) axis. The height of the terrace was approximately $2.290 \mathrm{~nm}$, which corresponded with the length of the molecules of $\mathrm{C}_{8}$-BTBT. Among various concentrations of the $\mathrm{C}_{8}$ BTBT solution, we found that $15 \mathrm{mg} / \mathrm{ml}$ was the optimized solution concentration. Next, we compared the electrical characteristics of the $\mathrm{C}_{8}$-BTBT transistors before and after solvent vapor annealing to determine if there were any improvement of the TFT performance in such areas as mobility, threshold voltage, and subthreshold swing. To investigate the effects of solvent vapor annealing, we calculated the contact resistance using a simultaneous extraction model. As a result, significantly lower contact resistances at small gate voltage range were observed when the device was treated with solvent vapor. This treatment induced better charge injection properties and led to smaller threshold voltages and smaller subthreshold swing. We suggest that solvent vapor annealing can be employed as a simple posttreatment to relieve the strain between layered contacts and solution-processed organic semiconducting films.

\section{Acknowledgments}

MJL would like to thank CW, HS, and KT for the valuable discussion and support for this work. This research was supported by the National Research Foundation (NRF) of Korea (2013K1A4A3055679, 2015R1A5A7037615,2016R1 A5A2B1010327).

\section{REFERENCES}

1. Y. Diao, B. Tee, G. Giri, J. Xu, and Z. Bao, "Solution Coating of Large-Area Organic Semiconductor Thin Films with Aligned Single-Crystalline Domains," Nat. Mater., 12 [7] 665-71 (2013).

2. T. Sakanoue and H. Sirringhaus, "Band-like Temperature Dependence of Mobility in a Solution-Processed Organic Semiconductor," Nat. Mater., 9 [9] 736-40 (2010).

3. K. Nakayama, Y. Hirose, J. Soeda, M. Yoshizumi, and E. Miyazaki, "Patternable Solution-Crystallized Organic Transistors with High Charge Carrier Mobility," Adv. Mater., 23 [14] 1626-29 (2011).

4. J. Smith, W. Zhang, R. Sougrat, K. Zhao, and T. Anthopoulos, "Solution-Processed Small Molecule-Polymer Blend Organic Thin-Film Transistors with Hole Mobility Greater than $5 \mathrm{~cm}^{2} / \mathrm{Vs}$," Adv. Mater., 24 [18] 2441-46 (2012).

5. J. Kim, J. Jeong, H. Cho, C. Lee, and Y. Hong, "All-Solution-Processed Bottom-Gate Organic Thin-Film Transistor with Improved Subthreshold Behaviour Using Functionalized Pentacene Active Layer,” J. Phys. D: Appl. Phys., 42 [11] 115107 (2009).

6. H. Sirringhaus, "Device Physics of Solution Processed Organic Field Effect Transistors," Adv. Mater., 17 [20] 2411-25 (2005).

7. M. Lee, "Inkjet Printing Technology Still in Progress," J. Korean Ceram. Soc., 48 [6], 543-48 (2011).

8. J. Kim, J. Lee, Y. Lee, W. Kwon, and Y. Kim, "Preparation and Characterization of Low K Thin Film Using A Preceramic Polymer," J. Korean Ceram. Soc., 48 [6] 499-503 (2011).

9. M. Lee, D. Gupta, N. Zhao, M. Heeney, and H. Sirringhaus, "Anisotropy of Charge Transport in a Uniaxially Aligned and Chain Extended, High Mobility, Conjugated Polymer Semiconductor," Adv. Funct. Mater., 21 [5] 932-40 (2011).

10. M. Lee, Z. Chen, R. Pietro, M. Heeney, and H. Sirringhaus, "Electrooptical Spectroscopy of Uniaxially Aligned Polythiophene Films in Field-Effect Transistors," Chem. Mater., 25 [10] 2075-82 (2013).

11. W. Pisula, A. Menon, M. Stepputat, I. Lieberwirth, and K. Müllen, "A Zone Casting Technique for Device Fabrication of Field Effect Transistors Based on Discotic Hexa Peri Hexabenzocoronene," Adv. Mater., 17 [6] 684-89 (2005).

12. C. Kim, S. Lee, E. Gomez, J. Anthony, and Y. Loo, "SolventDependent Electrical Characteristics and Stability of Organic Thin-Film Transistors with Drop Cast Bis (Triisopropylsilylethynyl) Pentacene," Appl. Phys. Lett., 93 [10] 103302 (2008).

13. Y. Yuan, G. Giri, A. Ayzner, A. Zoombelt, S. Mannsfeld, and Z. Bao, "Ultra-High Mobility Transparent Organic Thin Film Transistors Grown by an Off-Centre Spin-Coating Method," Nat. Commun., 5 1-9 (2014).

14. Y. Li, C. Liu, A. Kumatani, P. Darmawan, and K. Tsukagoshi, "Patterning Solution-Processed Organic Single-Crystal Transistors with High Device Performance," AIP Adv., 1 [2] 022149 (2011).

15. K. Dickey, J. Anthony, and Y. Loo, "Improving Organic Thin Film Transistor Performance through Solvent Vapor Annealing of Solution-Processable Triethylsilylethynyl Anthradith- 
iophene," Adv. Mater., 18 [13] 1721-26 (2006).

16. J. Wang, Z. Wu, J. Miao, K. Liu, and Y. Cao, "Solution-Processed Diketopyrrolopyrrole-Containing Small-Molecule Organic Solar Cells with 7.0\% Efficiency: In-Depth Investigation on the Effects of Structure Modification and Solvent Vapor Annealing," Chem. Mater., 27 [12] 4338-48 (2015).

17. J. Soeda, Y. Hirose, M. Yamagishi, A. Nakao, T. Uemura, and J. Takeya, "Solution-Crystallized Organic Field-Effect Transistors with Charge-Acceptor Layers: High-Mobility and Low-Threshold-Voltage Operation in Air," Adv. Mater.,
23 [29] 3309-14 (2011).

18. S. Lee, S. Jung, J. Lee, C. Kim, and M. Lee, "Investigation of Charge Injection Characteristics in Diketopyrrolopyrrole Ambipolar Semiconducting Polymers," Proc. SPIE., 9185 918512-1-8 (2014).

19. R. Di Pietro, D. Venkateshvaran, A. Klug, E. List-Kratochvil, and D. Neher, "Simultaneous Extraction of Charge Density Dependent Mobility and Variable Contact Resistance from Thin Film Transistors," App. Phys. Lett., 104 [19] 193501 (2014). 